\title{
SELF-SIMILARITY IN A COARSENING MODEL IN ONE DIMENSION
}

\author{
By \\ Jack Carr \\ and \\ Robert Pego
}

IMA Preprint Series \# 779

February 1991 


\title{
Self-Similarity in a Coarsening Model in One Dimension
}

\author{
Jack Carr \\ Department of Mathematics, Heriot-Watt University \\ Robert Pego \\ Department of Mathematics, University of Maryland, College Park
}

\begin{abstract}
Motivated from asymptotic laws of motion for transition layers in the equation $u_{t}=$ $\varepsilon^{2} u_{x x}+u-u^{3}$, we consider the following model for coarsening of a fine partition of an interval: Find the shortest subinterval of the partition, and joint it with its neighbors, combining three into one. Making a "random order assumption," we develop and study an unusual coagulation equation for the distribution of interval lengths. We establish the existence of a self-similar solution of this equation using Laplace transform techniques. Simulation data indicate that random order does persist if present initially, and the distribution approaches similarity form.
\end{abstract}

This work was supported by NSF grant DMS-8902422 and the SERC. Part of this work was completed at the Institute for Mathematics and its Applications, University of Minnesota. 


\section{$\S 1$. Introduction}

A process often occurring in phase separation is coarsening: The typical domain size grows in time as the larger domains grow at the expense of small domains, which shrink and disappear. Remarkably, it is often found that coarsening processes exhibit statistical self-similarity, in which, for example, the relative size distribution of domains may be constant in time, or the structure factor may scale in time in a self-similar fashion.

Motivated by a simple partial differential equation in one dimension whose solutions undergo a phase separation process (which will be discussed in the last section below), we consider the following simple deterministic model for nonconservative coarsening in one dimension. Let the line be subdivided initially by a large number of randomly placed points representing phase boundaries. We assume that this structure coarsens in discrete steps, as follows: the closest pair of phase boundaries annihilate each other, so that where there were $m$ phase boundaries before, there are $m-2$ phase boundaries afterward. In terms of the domains between boundaries, the smallest is eliminated, and merged with its two neighbors.

In this paper, we develop rate equations for the domain size distribution for this coarsening process, based on assumptions of the statistical randomness of the domains being merged. We then study self-similar growth in the domain size distribution. In sections 2 and 6 , we prove that the rate equations have a physically meaningful self-similar solution. Although an explicit formula is not available, we are able to characterize a number of properties of this solution. In section 3, through computer simulations of the coarsening process, we demonstrate that the similarity solution of the rate equations can act as an attractor for the process, which suggests that the statistical assumptions underlying the rate equations remain valid as coarsening proceeds (at least for the configurations considered).

The rate equations we develop are coagulation equations, and are related to some other models of coagulation which contain convolution terms describing the redistribution of mass, see [2], [6]. More distantly related is the Lifschitz-Slyozov-Wagner (LSW) theory of Ostwald ripening. See [9] for recent work on the LSW theory. Mathematically, our equations turn out to have some unusual features, in that they involve a free boundary problem for the smallest domain size as a function of number of domains destroyed, and incorporate a convolution term with spatial delay. The initial value problem for these equations is studied in sections 4 and 5 .

\section{§2. Derivation of the coagulation model}

To model the above coarsening process we let $f(x, t) d x$ denote the expected number of intervals per unit length, with length in $(x, x+d x)$ at time $t$. The smallest interval is denoted by $\mathcal{L}(t)$ so that $f(x, t)=0$ for $x<\mathcal{L}(t)$. We choose the time scale so that one merger per unit length occurs in unit time:

$$
f(\mathcal{L}(t), t) \frac{d \mathcal{L}}{d t} \quad=1
$$

The number of intervals per unit length is given by $N(t)$ :

$$
N(t)=\int_{0}^{\infty} f(x, t) d x
$$


The processes to be described by the rate equation are:

(a) formation of an interval of length $x$ by the coalescence of an interval of length $\mathcal{L}(t)$ with two intervals of length $y$ and $x-y-\mathcal{L}(t)$.

(b) the loss of an interval of length $x$ by any merger with another interval.

We make the statistical assumption that intervals undergoing coalescence (i.e. neighbors of intervals of length $\mathcal{L}(t))$ are distributed in the same way as intervals generally. Such an assumption has been called a "random order approximation" by Mullins. Under this assumption, the probability of (a) and (b) happening in time $d t$ are given by

$$
\frac{f(y, t)}{N(t)} \frac{f(x-y-\mathcal{L}(t), t)}{N(t)} d t \quad \text { and } \quad 2 \frac{f(x, t)}{N(t)} d t
$$

respectively. Using the above we obtain the rate equation for $f(x, t)$ :

$$
\frac{\partial f}{\partial t}(x, t)=N(t)^{-2}\left[\int_{0}^{\infty} f(y, t) f(x-y-\mathcal{L}(t), t) d y-2 f(x, t) N(t)\right]
$$

with $f(x, t)=0$ if $x<\mathcal{L}(t)$. Note that equations (2.1) and (2.2) remain invariant under the scaling $f \rightarrow a f, t \rightarrow t / a, a$ constant. With given initial shortest length $\mathcal{L}(0)=\mathcal{L}_{0}$ and initial distribution $f(x, 0)=\phi(x)$, equations (2.1) and (2.2) are to be solved for $f(x, t)$ and $\mathcal{L}(t)$.

It is interesting to note that the coagulation equation $(2.2)$ with $\mathcal{L}(t) \equiv 0$ appears in the Flory-Stockmayer theory of polymerization [6]. The character of the present model (2.1)(2.2) differs from previously studied coagulation models because of the free boundary at $x=\mathcal{L}(t)$. Existence and other basic properties of solutions of (2.1)-(2.2) will be established in later sections. In this section we seek a similarity solution.

Before doing this we obtain some information about moments of solutions of (2.1)(2.2). If $a(x)$ is a given function then formally

$$
\begin{gathered}
\frac{d}{d t} \int_{0}^{\infty} a(x) f(x, t) d x= \\
N(t)^{-2} \int_{0}^{\infty} \int_{0}^{\infty}(a(x+y+\mathcal{L}(t))-a(x)-a(y)-a(\mathcal{L}(t))) f(x, t) f(y, t) d x d y
\end{gathered}
$$

Taking $a(x)=1$ gives the evolution of $N(t)$, the total number of intervals per unit length. From (2.3), $d N(t) / d t=-2$ so that

$$
N(t)=N(0)-2 t .
$$

Taking $a(x)=x$ shows that the first moment is conserved:

$$
\int_{0}^{\infty} x f(x, t) d x=\rho
$$

Physically, this corresponds to the fact that in the processes (a) and (b) described above, length is neither created nor destroyed. 
We look for a similarity solution of (2.1)-(2.2) in the form

$$
f(x, t)=N(t)^{2} g(N x), \quad \mathcal{L}(t)=\alpha / N(t) .
$$

with $N$ given by (2.4), $\alpha$ a constant and with $g(w)=0$ for $w<\alpha$. This choice of scaling is motivated by the conservation of length in (2.5). Using $d N(t) / d t=-2$ and writing $w=N(t) x$,

$$
\begin{aligned}
f_{t}(x, t) & =-4 N(t) g(w)-2 N^{2}(t) x g^{\prime}(w)=-2 N(t)\left[2 g(w)+w g^{\prime}(w)\right], \\
N(t) & =N(t)^{2} \int_{0}^{\infty} g(N(t) x) d x=N(t) \int_{0}^{\infty} g(w) d w,
\end{aligned}
$$

so that

$$
\int_{0}^{\infty} g(w) d w=1
$$

Also

$$
\int_{0}^{\infty} f(y, t) f(x-y-\mathcal{L}(t), t) d y=N(t)^{3} \int_{0}^{\infty} g(y) g(w-y-\alpha) d y .
$$

Hence

$$
2(w g(w))^{\prime}+\int_{0}^{\infty} g(y) g(w-y-\alpha) d y=0
$$

Integrating this equation, assuming that $g$ is integrable with $w g(w) \rightarrow 0$ as $w \rightarrow \infty$, and using (2.6) we get that $2 \alpha g(\alpha)=1$. Let $h(w)$ be the solution of (2.7) with $\alpha=1$ so that $h(w)=0$ for $w<1$ and $h$ satisfies

$$
2(w h(w))^{\prime}+\int_{0}^{\infty} h(y) h(w-y-1) d y=0, \quad w \geq 1, \quad h(1)=1 / 2 .
$$

Then $g(w)=h(w / \alpha) / \alpha$ satisfies (2.7). Since $h(w)=0$ for $w<1$, the lower and upper integration limits in the convolution term in $(2.8)$ are 1 and $\max (1, w-2)$ respectively. So this term depends only on values of $h(y)$ for $y$ less than $w-2$, and the solution may be generated by successive integrations. In particular, the convolution term is zero for $1 \leq w \leq 3$ so that

$$
h(w)=1 / 2 w, \quad 1 \leq w \leq 3 .
$$

Writing $K(w)=h(w+1)$, it follows $K(w)=1 / 2(1+w)$ for $0 \leq w \leq 2$ and

$$
K(w+2)=\frac{1}{2(w+3)}\left(1-\int_{0}^{w} \int_{0}^{y} K(z) K(y-z) d z d y\right) \quad \text { for } w \geq 0 .
$$

For the numerical results in section 3, we determine $h(w)$ from these equations using a straightforward trapezoid rule discretisation and extrapolation.

It remains to show that $h$ defined by (2.8) is positive with $h(w) \rightarrow 0$ sufficiently rapidly as $w \rightarrow \infty$ so that $\rho_{1}=\int_{0}^{\infty} w h(w) d w$ is finite. This is necessary in order to demonstrate that a physically meaningful similarity solution exists, and will be done in section 6 . In 
section 6 we also compute that $\rho_{1}=2 e^{\gamma}=3.562145 \ldots$, where $\gamma$ is Euler's constant. Given a solution $g(w)=h(w / \alpha) / \alpha$ of (2.7), then we find that $\int_{0}^{\infty} w g(w) d w=\alpha \rho_{1}$. Thus, given $\rho>0$, there is a unique similarity solution with first moment $\rho$, namely $g(w)=h(w / \alpha) / \alpha$ with $\alpha=\rho / \rho_{1}$.

\section{$\S 3$. Simulations}

We will exhibit simulation data for the discrete coarsening process described in the introduction, corresponding to three different initial distributions of interval lengths. To make the simulation finite, we replace the line by a circle of fixed length. We construct a subdivision of this circle, and coarsen this structure by discrete steps, joining the smallest interval with its two neighbors. The initial configuration is constructed by choosing successive interval lengths randomly from a probability density $\phi(x)$. We consider:
(a) $\phi(x)=e^{-x}$
(Poisson distribution with mean 1)
(b) $\phi(x)= \begin{cases}1 / 2, & 1 \leq x \leq 3 \\ 0 & \text { otherwise }\end{cases}$
(Uniform distribution on $[1,3]$ )
(c) $\phi(x)=\left\{\begin{array}{lll}\lambda e^{-\lambda(x-a)} & x \geq a & \text { (Add } a \text { to a length chosen from the } \\ 0 & x<a & \text { Poisson distribution with mean } 1 / \lambda)\end{array}\right.$

In case (c) we take $\lambda$ and $a$ to provide a close fit to the similarity solution $h(x)$. Motivated by the analysis in section 6 below, we take $\lambda=0.37250741, a=\lambda^{-1} \ln 2-1=0.86076078$. We start with $10^{5}$ intervals in each simulation. When $55 \%, 75 \%$ and $95 \%$ of the intervals have been destroyed, we plot the distribution function for relative length, averaged over 20 runs. To be precise, we fix a bin width $\delta(=.04, .06, .1$ respectively $)$, and let $w_{j}=j \delta$, $j=0,1, \ldots$. Let $M$ be the ratio of the total number of intervals \# $(t)$ at the current time to the total initial number $\#_{0}=10^{5}$. For $M=.45, .25, .05$ we plot $g_{j}$ vs. $w_{j}$, where

$$
g_{j}=\left(\# \text { of intervals whose length } x \text { satisfies } w_{j} \leq M x \leq w_{j+1}\right) /\left(\delta \#_{0}\right)
$$

Note that $\left\{g_{j}\right\}$ is a discrete probability density, with

$$
\delta \sum_{j=0}^{\infty} g_{j}=1, \quad \delta \sum_{j=0}^{\infty} w_{j} g_{j}=(\text { total length }) / \#_{0}+O(\delta)=\rho_{0}+O(\delta)
$$

where $\rho_{0}$ is a constant. To compare these data to solutions of (2.1), (2.2), we suppose $f(x, 0)=\phi(x)$ is normalized so that $\int_{0}^{\infty} \phi(x) d x=N_{0}=1$. Then $M$ corresponds to $N(t)=$ $1-2 t$, and, with $f(x, t)=N(t)^{2} g(N(t) x, t), g_{j}$ corresponds to $\delta^{-1} \int_{w_{j}}^{w_{j}+\delta} g(w, t) d w \approx$ $g\left(w_{j}, t\right)$. Also $\rho_{0}$ corresponds to $\rho=\int_{0}^{\infty} w g(w, t) d w=\int_{0}^{\infty} x \phi(x) d x$. Corresponding to the various cases, we have (a) $\rho=1$, (b) $\rho=2$, (c) $\rho=3.545272$.

The data appear in figures 1-3, corresponding to cases (a)-(c) respectively. In figures $1 \mathrm{a}, 2 \mathrm{a}$, and $3 \mathrm{a}$, we plot $g_{j}$ vs. $w_{j}$ for $M=.45$ (solid), .25 (dashed), and .05 (dotted). In 
figures $1 \mathrm{~b}, 2 \mathrm{~b}$, and $3 \mathrm{~b}$ we compare the last simulation data to the similarity solution. The markers plot $g_{j}$ vs. $w_{j}$ for $M=.05$, while the solid curve plots $\delta^{-1} \int_{w_{j}}^{w_{j}+\delta} g(w) d w$ vs. $w_{j}$, where $g$ is the similarity solution $g(w)=h(w / \alpha) / \alpha$. Here $\alpha=\rho / \rho_{1}$ is as appropriate to each case: (a) $\alpha=.28072974$, (b) $\alpha=.56145948$, (c) $\alpha=.99526305$.

The results indicate convergence to the similarity solution of $(2.1),(2.2)$ in all cases. This provides evidence that the "random order assumption" made in deriving equations (2.1), (2.2) is valid for the configurations studied. Convergence is quickest in case (c), where $\phi(x)$ most resembles $g(x)$, and slowest in case (b), where the discrete probability density $g_{j}$ initially develops oscillations, which are eventually "forgotten" once most of the intervals have been destroyed.

\section{$\S 4$. Existence of solutions}

In this section and the next we prove some rigorous results concerning existence, regularity and infinite propagation speed for solutions of equations (2.1)-(2.2). The results are most easily stated in terms of the (left) inverse function to $\mathcal{L}(t)$, which we denote by $T(x)$, satisfying $T(\mathcal{L}(t))=t$, or

$$
\frac{d T}{d x}=f(x, T(x))
$$

This will allow for the possibility that gaps occur in the number density, so that perhaps $f(x, t)=0$ for $x$ in some interval and $\mathcal{L}(t)$ experiences a jump discontinuity. Below we denote by $B\left(\mathrm{R}^{+}\right)$the space of bounded measurable functions on $[0, \infty)$.

Theorem 4.1. (Basic existence and uniqueness) Assume $\mathcal{L}_{0}>0$ and $N(t)=N_{0}-2 t$ are given. Assume $\phi \in B\left(\mathbb{R}^{+}\right)$with $\phi(x) \geq 0$ for $x \geq \mathcal{L}_{0}, \phi(x)=0$ for $x<\mathcal{L}_{0}$, and

$$
\int_{0}^{\infty} \phi(x) d x=N_{0}<\infty, \int_{0}^{\infty} x \phi(x) d x=\rho<\infty .
$$

Then there exists a unique solution to (2.1)-(2.2) with $f(x, 0)=\phi(x)$ in the following sense: There exists a nonnegative locally Lipschitz function $F:\left[0, N_{0} / 2\right] \rightarrow B\left(\mathbb{R}^{+}\right)$and a unique nondecreasing Lipschitz function $T(x)$ on $[0, \infty)$ such that

$$
f(x, t)=F(x, t) \quad \text { for } t \leq T(x), \quad f(x, t)=0 \quad \text { for } t>T(x),
$$

(4.1) holds for a.e. $x>0$, and (2.2) holds for all $x>0$, for a.e. $t<T(x)$, where $\mathcal{L}(t)$ is any inverse function for $T$. Moreover, we have

$$
N(t)=\int_{0}^{\infty} f(x, t) d x, \quad \int_{0}^{\infty} x f(x, t) d x=\rho, \text { for } 0 \leq t<N_{0} / 2,
$$

and $T(x) \rightarrow N_{0} / 2$ as $x \rightarrow \infty$, so $\mathcal{L}(t) \rightarrow \infty$ as $t \rightarrow N_{0} / 2$.

Proof. We construct $F(x, t)$ and $T(x)$ inductively for $0 \leq t<N_{0} / 2$ and $x \leq \mathcal{L}_{k}=$ $(2 k+1) \mathcal{L}_{0}, k=0,1, \ldots$. These functions will satisfy

$$
(F(x, t) / N(t))_{t}=S(x-3 \mathcal{L}(t), t) N(t)^{-3}, \quad F(x, 0)=\phi(x)
$$


where

$$
S(x, t)=\int_{0}^{x} F(\mathcal{L}+y, t) F(\mathcal{L}+x-y, t) d y \geq 0, \quad S(x, t)=0 \quad \text { for } x<0 .
$$

and

$$
T^{\prime}(x)=F(x, T(x)), \quad x>\mathcal{L}_{0}, \quad T\left(\mathcal{L}_{0}\right)=0 .
$$

With (4.2), this system of equations is equivalent to (2.1)-(2.2). To proceed, for $0 \leq t<$ $N_{0} / 2, x<\mathcal{L}_{1}=3 \mathcal{L}_{0}$, solve

$$
(F(x, t) / N(t))_{t}=0
$$

so $F(x, t)=N(t) \phi(x) / N_{0}$. From (4.5), $T(x)$ is now determined for $x \leq \mathcal{L}_{1}$. $F$ is bounded and nonnegative, so $T$ is Lipschitz and nondecreasing; in fact

$$
T(x)=\left(N_{0} / 2\right)\left[1-\exp \left(-2 N_{0}^{-1} \int_{0}^{x} \phi(y) d y\right)\right],
$$

so it is clear that $T(x)<N_{0} / 2$ for $x \leq \mathcal{L}_{1}$. Choose $\mathcal{L}(t)$ to be any right inverse for $T(x)$, so $\mathcal{L}(t)$ is defined and increasing for $0 \leq t \leq T\left(\mathcal{L}_{1}\right)$.

Now proceed to the induction step. Assume $F(x, t) \geq 0$ and $T(x)$ nondecreasing are known for $x \leq \mathcal{L}_{k}, 0 \leq t<N_{0} / 2$, and $\mathcal{L}(t)$ is known for $0 \leq t \leq T\left(\mathcal{L}_{k}\right)$. Then $S(x, t)$ in (4.4) is defined when $\mathcal{L}(t)+x \leq \mathcal{L}_{k}, 0 \leq t \leq T\left(\mathcal{L}_{k}\right)$, and is bounded, measurable and nonnegative. Hence $S(x-3 \mathcal{L}(t), t)$ in (4.3) is known for $x \leq \mathcal{L}_{k}+2 \mathcal{L}(t)$; in particular, for $x \leq \mathcal{L}_{k}+2 \mathcal{L}_{0}=\mathcal{L}_{k+1}$. Solving (4.3) for $0 \leq t \leq T\left(\mathcal{L}_{k}\right),(4.6)$ for $t>T\left(\mathcal{L}_{k}\right), F(x, t)$ is determined for $x \leq \mathcal{L}_{k+1}, 0 \leq t<N_{0} / 2$. $F$ is nonnegative, Lipschitz in $t$ for each $x$, and measurable. From (4.5), $T(x)$ is now determined for $x \leq \mathcal{L}_{k+1}$ with $T$ Lipschitz and nondecreasing. Noting that for $\mathcal{L}_{k} \leq x \leq \mathcal{L}_{k+1}, t \geq T\left(\mathcal{L}_{k}\right)$, either $x-3 \mathcal{L}(t)<0$ or $t \geq T\left(\mathcal{L}_{k}\right),(4.6)$ is satisfied, so a short computation gives

$$
N_{0}-2 T(x)=\left(N_{0}-2 T_{k}\right) \exp \left(-2 N\left(T_{k}\right)^{-1} \int_{\mathcal{L}_{k}}^{\infty} F\left(y, T_{k}\right) d y\right)
$$

where $T_{k}=T\left(\mathcal{L}_{k}\right)$. Thus, $T(x)<N_{0} / 2$ for $x \leq \mathcal{L}_{k+1}$.

At this point $F(x, t)$ and $T(x)$ are defined for all $x \geq 0,0 \leq t<N_{0} / 2$. Since $T(x)$ is nondecreasing, $T_{\infty}=\lim _{x \rightarrow \infty} T(x)$ exists with $T_{\infty} \leq N_{0} / 2 . \mathcal{L}(t)$ is defined for $0 \leq t<T_{\infty}$. In order to complete the proof, we establish the following results. Below, $f(x, t)$ is defined by (4.2).

Lemma 4.3. Let $a$ be a continuous function, $0 \leq t<T_{\infty}$, and $A \geq \mathcal{L}(t)$. Then

$$
\int_{0}^{A} a(x) f(x, t) d x=\int_{0}^{A} a(x) \phi(x) d x+\int_{0}^{t} P(s) N(s)^{-2} d s
$$

where, with $\mathcal{L}=\mathcal{L}(s)$,

$$
\begin{aligned}
P(s)=\int_{D(s)} & a(y+z+\mathcal{L}) f(y, s) f(z, s) d y d z-z N(s) \int_{0}^{A} a(y) f(y, s) d y \\
& -N(s)^{2} a(\mathcal{L}(s)) .
\end{aligned}
$$


Here $D(s)=\{(w, z): w \geq 0, z \geq 0, w+z \leq A-\mathcal{L}(s)\} \subseteq[0, A] \times[0, A]$.

The identity above corresponds to the formal identity (2.3). The next lemma establishes the validity of this identity for $a(x)=1$ or $x$.

Lemma 4.4. For $0 \leq t<T_{\infty}$ we have
i) $N(t)=N_{0}-2 t=\int_{0}^{\infty} f(x, t) d x$,
ii) $\int_{0}^{\infty} x f(x, t) d x=\rho \quad$ independent of $t$,
iii) $\mathcal{L}(t) N(t)<\rho$.

Corollary 4.5. $\lim _{x \rightarrow \infty} T(x)=N_{0} / 2$, so $\mathcal{L}(t)$ is defined for $0 \leq t<N_{0} / 2$ and $\lim _{t \rightarrow N_{0} / 2} \mathcal{L}(t)=\infty$

Lemma 4.6. For $0 \leq t<N_{0} / 2$, we have

i) $\sup _{x} F(x, t) \leq \sup _{x} \phi(x)\left(N(t) / N_{0}\right)^{1 / 2}$,

ii) $\sup _{x}|\partial F / \partial t| \leq \sup _{x} F(x, t) / N(t)$.

Proof of Lemma 4.3. Multiply (2.2) by $a(x)$ and integrate from 0 to $\min (T(x), t)$ in $t$, from 0 to $A$ in $x$. The left hand side yields, by (4.5),

$$
\begin{aligned}
& \int_{0}^{A} \int_{0}^{\min (T(x), t)} a(x) \frac{\partial f}{\partial t}(x, s) d s d x= \\
& \int_{0}^{\mathcal{L}(t)} a(x)(f(x, T(x))-\phi(x)) d x+\int_{\mathcal{L}(t)}^{A} a(x)(f(x, t)-\phi(x)) d x \\
= & \int_{0}^{t} a(\mathcal{L}(s)) d s+\int_{0}^{A} a(x)(f(x, t)-\phi(x)) d x,
\end{aligned}
$$

using the change of variables $s=T(x)$ in the first term. After interchanging the order of integration, the right hand side yields $\int_{0}^{t} Q(s) N(s)^{-2} d s$, where

$$
\begin{aligned}
Q(s) & =\int_{\mathcal{L}}^{A}\left[-2 N(s) f(x, s)+\int_{\mathcal{L}}^{x-2 \mathcal{L}} f(y, s) f(x-y-\mathcal{L}, s) d y\right] a(x) d x \\
& =-2 N(s) \int_{0}^{A} a(y) f(y, s) d y+\int_{D(s)} a(y+z+\mathcal{L}) f(y, s) f(z, s) d y d z
\end{aligned}
$$

The result follows.

Proof of Lemma 4.4. For part i), put $a(x)=1$ in Lemma 4.3. Note that

$$
\int_{D(s)} f(y, s) f(z, s) d y d z \leq\left(\int_{0}^{A} f(y, s) d y\right)^{2} .
$$


Then if we define $b(t)=\int_{0}^{A} f(y, t) d y-N(t)$, we find that $b$ is Lipschitz, $b(0) \leq 0$ and

$$
\begin{aligned}
b^{\prime}(t) & =\frac{d}{d t} \int_{0}^{A} f(y, t) d y+2 \leq N^{-2}\left((b+N)^{2}-2 N(b+N)-N^{2}\right)+2 \\
& =b^{2} / N^{2}
\end{aligned}
$$

Since $b$ is bounded, Gronwall's inequality implies that $b(t) \leq 0$ for all $t<T(A)$. Hence we find that

$$
\int_{0}^{\infty} f(x, t) d x \leq N(t) \quad \text { for } 0 \leq t<T_{\infty}
$$

by letting $A$ tend to infinity. To prove equality in (4.8), we let $A \rightarrow \infty$ in Lemma 4.3, using Fubini's theorem to obtain

$$
\lim _{A \rightarrow \infty} \int_{D(s)} f(y, x) f(z, s) d y d z=\left(\int_{0}^{\infty} f(x, s) d x\right)^{2}
$$

Hence with $b(t)=\int_{0}^{\infty} f(y, t) d y-N(t)$ we have $b(0)=0$ and $b$ is bounded and measurable, with

$$
\begin{aligned}
b(t) & =\int_{0}^{t} N(s)^{-2}\left((b+N)^{2}-2 N(b+N)-N^{2}\right)+2 d s \\
& =\int_{0}^{t} b(s)^{2} / N(s)^{2} d s .
\end{aligned}
$$

Bootstrapping, it follows that $b$ is Lipschitz, hence smooth, with $b^{\prime}(t)=b^{2} / N^{2}, b(0)=0$. Hence $b(t)=0$ for all $t$, which proves $\mathrm{i}$ ).

Part ii) is proved in a similar fashion. Choose $a(x)=x$. Then we have, using part i),

$$
\begin{aligned}
\int_{D(s)}(y+z+\mathcal{L}(s)) f(y, s) f(z, s) d y d z \leq \\
2 N(s) \int_{0}^{A} x f(x, s) d x+\mathcal{L}(s) N(s)^{2} .
\end{aligned}
$$

Hence, if $b(t)=\int_{0}^{A} x f(x, t) d x$, we have $b(0) \leq \rho$,

$$
b^{\prime}(t) \leq N^{-2}\left(2 N b+\mathcal{L} N^{2}-2 N b-\mathcal{L} N^{2}\right)=0
$$

Hence $b(t) \leq \rho$ for $0 \leq t<T(A)$, hence $\int_{0}^{\infty} x f(x, t) d x \leq \rho$. Putting $b(t)=\int_{0}^{\infty} x f(x, t) d x$ and letting $A \rightarrow \infty$ in Lemma 4.3, it follows $b(t)=\rho+\int_{0}^{t} P(s) N(s)^{-2} d s$ where $P(s)=0$, proving ii).

Part iii) is proved as follows:

$$
\rho=\int_{0}^{\infty} x f(x, t) d x=\int_{\mathcal{L}(t)}^{\infty} x f(x, t) d x>\int_{0}^{\infty} \mathcal{L}(t) f(x, t) d x=\mathcal{L}(t) N(t)
$$


It follows then that $x N(T(x))<\rho$ which implies $\lim _{x \rightarrow \infty} N(T(x))=0$ or $\lim _{x \rightarrow \infty} T(x)=$ $N_{0} / 2$, yielding Corollary 4.5 .

Proof of Lemma 4.6. From the definition of $S(x, t)$ in (4.4), and (4.8), we have $S(x, t) \leq$ $N(t) \sup _{x} f(x, t)$. Hence from (4.3) follows

$$
F(x, t) / N(t) \leq \phi(x) / N_{0}+\int_{0}^{t} \sup _{y} F(y, x) N(s)^{-2} d s \stackrel{\text { def }}{=} V(t) .
$$

Thus we have $N V^{\prime} \leq V$ and integration yields $V(t) \leq V(0)\left(N_{0} / N(t)\right)^{1 / 2}$ from which part i) follows. Part ii) follows from (4.3) and the bound on $S(x, t)$ above.

The remaining assertions of Theorem 4.1 follow from the results 4.3-4.6. In particular, the bounds in Lemma 4.6 ensure that $F$ is locally Lipschitz as a map from $\left[0, N_{0} / 2\right)$ to $B\left(\mathrm{R}^{+}\right)$. This concludes the proof of Theorem 4.1 .

\section{$\S 5$. Instantaneous positivity and $C^{1}$ solutions}

Proposition 5.1. Make the hypotheses of Theorem 4.1. Assume $\phi(x)>0$ for $\mathcal{L}_{0} \leq$ $x \leq 3 \mathcal{L}_{0}$. Then $f(x, t)>0$ for all $x \geq \mathcal{L}_{0}, 0<t \leq T(x)$. Furthermore, $T(x)$ is strictly increasing for $x \geq \mathcal{L}_{0}$, so $\mathcal{L}(t)$ is uniquely defined and locally Lipschitz.

Proof. By following the proof of Theorem 4.1, we will prove by induction on $k$ that

$$
F(x, t)>0 \quad \text { for } \mathcal{L}_{0} \leq x \leq \mathcal{L}_{k}, \quad 0<t
$$

For $k=1$, this follows from (4.6) and the hypothesis on $\phi$. Then (4.5) implies that $T(x)$ is strictly increasing for $\mathcal{L}_{0} \leq x \leq \mathcal{L}_{1}$, so $\mathcal{L}(t)$ is continuous for $0 \leq t \leq T\left(\mathcal{L}_{1}\right)$. Now suppose that (5.1) holds for some $k \geq 1$. Suppose $\mathcal{L}_{k}<x \leq \mathcal{L}_{k+1}$, and suppose $t_{0}>0$ is so small that $x>3 \mathcal{L}(t)$ for $0<t<t_{0}$. Then for $y \in\left[0, \mathcal{L}_{k}-\mathcal{L}(t)\right]$ we have $F(\mathcal{L}+y, t)>0$, and for $y \in[0, x-3 \mathcal{L}]$ we have $\mathcal{L} \leq x-2 \mathcal{L}-y \leq x-2 \mathcal{L}_{0} \leq \mathcal{L}_{k}$, so $F(x-2 \mathcal{L}-y, t)>0$. It follows that $S(x-3 \mathcal{L}(T), t)>0$ for $0<t<t_{0}$, and so (4.3) implies $F(x, t)>0$ for $t>0$, i.e. (5.1) holds with $k$ replaced by $k+1$.

Theorem 5.2. In addition to the hypotheses of Theorem 4.1 , assume that $\phi \in C^{1}\left(\left[\mathcal{L}_{0}, \infty\right)\right)$ and $\phi(x)>0$ for $\mathcal{L}_{0} \leq x \leq 3 \mathcal{L}_{0}$. Then the solution $f \in C^{1}(\bar{Q})$, where $Q=\{(x, t) \mid x>$ $\mathcal{L}(t), t>0\}$. the function $\mathcal{L} \in C^{2}\left(\left[0, N_{0} / 2\right)\right)$, and (2.1)-(2.2) hold for all $(x, t) \in Q$.

Remark. Even if the initial data are smooth, the solution cannot be expected to be everywhere more regular than indicated in this result. It appears that the solution will be only piecewise smooth at best, with a complex pattern of weak singularities - curves across which some derivative of $f$ suffers a discontinuity.

Proof. We will prove by induction on $k$ that

$$
\begin{aligned}
& F(x, t) \text { is } C^{1} \quad \text { for } \mathcal{L}_{0} \leq x \leq \mathcal{L}_{k}, \quad 0 \leq t<N_{0} / 2 \text {, } \\
& \mathcal{L}(t) \text { is } C^{2} \text { for } 0 \leq t \leq T\left(\mathcal{L}_{k}\right) \text {. }
\end{aligned}
$$

That (5.2) is true for $k=1$ follows from (4.6). Then (4.5) implies $T(x)$ is $C^{2}$ for $\mathcal{L}_{0} \leq$ $x \leq \mathcal{L}_{1}$ with $d T / d x>0$, so (5.3) is true with $k=1$. Assume that (5.2) and (5.3) hold for 
some $k \geq 1$. First we claim that $S(x-3 \mathcal{L}(t), t)$ is $C^{1}$ for $3 \mathcal{L}(t) \leq x \leq \mathcal{L}_{k+1}=\mathcal{L}_{k}+2 \mathcal{L}_{0}$, $0 \leq t<N_{0} / 2$. For $0 \leq x \leq \mathcal{L}_{k+1}-3 \mathcal{L}(t)$, put $u(x, t)=F(\mathcal{L}(t)+x, t)$. By (5.2), (5.3) it follows that $u$ is $C^{1}$ for $0 \leq x \leq \mathcal{L}_{k+1}-3 \mathcal{L}(t)$. But $S(x, t)=\int_{0}^{x} u(x-y, t) u(y, t) d y$, so it follows by explicit differentiation that $S$ is $C^{1}$ for $0 \leq x \leq \mathcal{L}_{k+1}-3 \mathcal{L}(t)$, which proves the first claim.

Next, put $u(x, t)=S(x-3 \mathcal{L}(t), t)$. Then

$$
\frac{F(x, t)}{N(t)}-\frac{\phi(x)}{N_{0}}= \begin{cases}\int_{0}^{T(x / 3)} u(x, s) d s & \text { for } x \leq 3 \mathcal{L}(T) \\ \int_{0}^{t} u(x, s) d s & \text { for } x \geq 3 \mathcal{L}(T)\end{cases}
$$

Now one may verify that $\partial F / \partial x$ is continuous for $\mathcal{L}_{0} \leq x \leq \mathcal{L}_{k+1}$ by explicit differentiation, using the fact that when $t=T(x / 3), 3 \mathcal{L}(t)=x$, so $u(x, t)=S(0, t)=0$. Together with (4.3), this establishes (5.2), and (5.3) follows from (4.5) and the fact that $F>0$. This finishes the proof.

\section{$\S 6$. Existence of the similarity solution}

It is convenient to write $(2.8)$ in the form

$$
2[(1+x+2) K(x+2)]^{\prime}+\int_{0}^{x} K(y) K(x-y) d y=0, \quad x>0
$$

with $K(x)=h(x+1)$. We first derive a crude bound for $K$.

Lemma 6.1. For $x \geq 0, \quad|2 K(x)| \leq e^{x / 4}$.

Proof. This is true for $0 \leq x \leq 2$ since $2 K(x)=(1+x)^{-1}$ there by (2.9). From (6.1),

$$
|2 K(x+2)|=\frac{1}{(x+3)} \int_{0}^{x} \int_{0}^{z} K(y) K(z-y) d y d z
$$

so that

$$
|2 K(x+2)| \leq \frac{1}{4(x+3)} \int_{0}^{x} \int_{0}^{z} z e^{z / 4} d z \leq \exp ((x+2) / 4) .
$$

Corollary 6.2. The Laplace transform of $K, \quad \beta(z)=\int_{0}^{\infty} e^{-z x} K(x) d x$, is well defined for $z>1 / 4$.

Using $2(1+x) K(x)=1$ for $0 \leq x \leq 2$ and taking the Laplace transform of (6.1) we get $e^{2 z}\left(-1+2 z\left(\beta-\beta^{\prime}\right)\right)=-\beta^{2}$. Writing $u(z)=e^{-z} \beta(z)$ so that $u(z)$ is the Laplace transform of $h$,

$$
2 z e^{z} u^{\prime}(z)=u^{2}-1
$$

Using the boundary condition $u(z) \rightarrow 0$ as $z \rightarrow \infty$, we find that

$$
u(z)=\tanh \left(E_{1}(z) / 2\right), \quad E_{1}(z)=\int_{z}^{\infty} y^{-1} e^{-y} d y .
$$


where $E_{1}(z)$ is the exponential integral [1],[8]. To show that $K(x)>0$ it is sufficient to show that $u(z)$ is the Laplace transform of a positive function. We require the following.

Definition. Let $f:[a, \infty) \rightarrow \mathbf{R}$ be $C^{\infty}$ on $(a, \infty)$ and continuous at $a$. Then $f$ is said to be completely monotone on $[a, \infty)$ if

$$
(-1)^{k} f^{(k)}(z) \geq 0, \quad k=0,1, \ldots, \quad a<z<\infty .
$$

As examples, $(1+z)^{-1}$ and $e^{-z}$ are completely monotone. We will use the following result due to Bernstein [10, p.160]:

Theorem 6.3. If $f$ is completely monotone in $[0, \infty)$ then

$$
f(z)=\int_{0}^{\infty} e^{-z t} d \alpha(t)
$$

where $\alpha(t)$ is bounded and non-decreasing.

To prove that $u(z)$ is completely monotone we make use of the following criterion [7, p.441]:

Lemma 6.4. If $f$ and $g$ are completely monotone then so is $f g$. If $f$ is completely monotone and $\psi>0$ with $\psi^{\prime}$ completely monotone then $f(\psi(z))$ is completely monotone. In particular, $\exp (-\psi(z))$ is completely monotone.

Lemma 6.5. $u(z)$ is completely monotone on $[0, \infty)$.

Proof. It is sufficient to show that

$$
T(z)=(u(z)+1) / 2=\left[1+\exp \left(-E_{1}(z)\right)\right]^{-1}
$$

is completely monotone. Since $(1+z)^{-1}$ is completely monotone, by Lemma 6.4 it is enough to show that $\exp \left(-E_{1}(z)\right)^{\prime}$ is completely monotone. Now

$$
\left(\exp \left(-E_{1}(z)\right)\right)^{\prime}=-E_{1}^{\prime}(z) \exp \left(-E_{1}(z)\right)=\exp \left(-E_{1}(z)-\ln z-z\right) .
$$

Now by Lemma 6.4 it is sufficient to show that $\left(E_{1}(z)+\ln z+z\right)^{\prime}$ is completely monotone. This follows from

$$
\left(E_{1}(z)+\ln z+z\right)^{\prime}=z^{-1}-z^{-1} e^{-z}+1=\int_{0}^{1} e^{-t z} d t+1 .
$$

Corollary 6.6. The unique solution of equation (2.8) satisfies $h(w)>0$ for all $w \geq 1$.

Proof. It follows from Lemma 6.5 and Theorem 6.3 that $h$ is nonnegative. If $h(w)=0$ for some minimal $w>1$, then $(2.8)$ implies $h^{\prime}(w)<0$. The result follows.

The asymptotic behaviour of the similarity solution $h(x)$ as $x \rightarrow \infty$ depends on the poles of its Laplace transform given by (6.2). Write $\sigma(z)=\exp \left(-E_{1}(z)\right), u(z)=$ $(1-\sigma(z)) /(1+\sigma(z))$. From [8], the exponential integral may be written in the form

$$
E_{1}(z)=-\ln z-\gamma+\int_{0}^{z}\left(1-e^{-y}\right) y^{-1} d y
$$


where $\gamma$ is Euler's constant. Since the integral in (6.3) is entire, it follows that $\sigma(z)$ is an entire function of $z$ so we have to study the zeros of $\sigma(z)+1$. For real $z, \sigma(z)$ is an increasing function of $z$, with $\sigma(z) \rightarrow-\infty$ as $z \rightarrow-\infty, \sigma(0)=0$ and $\sigma(z) \rightarrow 1$ as $z \rightarrow \infty$. Hence $u(z)$ has a unique real pole at $z=-\lambda$, where numerically, $\lambda=.372507410781$ to 12 decimal places. Since $u(z)$ is the Laplace transform of a positive function, the axis of convergence of $u(z)$ has a real singularity [10, p.58]. Thus the integral $u(z)=\int_{0}^{\infty} h(w) e^{-w z} d w$ converges for $\operatorname{Re}(z)>-\lambda$. The asymptotic behavior of $h(x)$ is determined by the residue of $u(z)$ at $z=-\lambda$ by:

Theorem 6.7. Define $\eta=\lim _{z \rightarrow-\lambda}(z+\lambda) u(z)=2 \lambda e^{-\lambda} \approx .5133186$. Then

$$
\lim _{x \rightarrow \infty} e^{\lambda x} h(x)=\eta \text {. }
$$

Proof. The result follows immediately from Ikehara's theorem [10] as modified by Diekmann and Kaper [5], once we show that $u(z)$ has no singularities for $\operatorname{Re}(z)=-\lambda$ other than the simple pole at $z=-\lambda$. Since $\sigma(-\lambda)=-1$, it follows from (6.3) that $E_{1}(-\lambda \pm i 0)=\mp i \pi$. Thus if $\sigma(-\lambda-i \lambda t)=-1$ for some $t \in \mathbf{R}$, then

$$
\int_{-\lambda}^{-\lambda-i t \lambda} y^{-1} e^{-y} d y=2 k \pi i
$$

for some integer $k$. Taking real and imaginary parts in (6.4), it follows that $\sigma(-\lambda-i \lambda t)=$ -1 for some $t \in \mathbb{R}$ if and only if

$$
A(t)=0, \quad B(t)=2 k \pi i
$$

where

$$
A(t)=e^{\lambda} \int_{0}^{t} \frac{(-\sin \lambda \theta+\theta \cos \lambda \theta)}{1+\theta^{2}} d \theta, \quad B(t)=e^{\lambda} \int_{0}^{t} \frac{(\cos \lambda \theta+\theta \sin \lambda \theta)}{1+\theta^{2}} d \theta .
$$

Note that $A$ is even, and $B$ is odd in $t$. The Theorem now follows from the following lemma. We thank Peter Bates for help with its proof.

Lemma 6.8. $0<B(t)<2 \pi$ for $t>0$.

Proof. Consider $B$ as a function of both $t$ and $\lambda$. Then $B(t, 0)=\arctan t$ and $\partial B / \partial \lambda(t, \lambda)=e^{\lambda} \sin (\lambda t) / \lambda$. So $B(t)=\arctan t+C(t)$ where $C(t)=\int_{0}^{\lambda} s^{-1} e^{s} \sin s t d s$. Since $0<\arctan t<\pi / 2$ for $t>0$, it suffices to show that $0<C(t)<3 \pi / 2$ for $t>0$. To proceed with the proof we need the estimate $1 / 3<\lambda<\log (3 / 2)$, which is clear from tables [1]. Now to conclude the proof, we have two cases:

(i) $t<\pi / \lambda$. Since $0<\sin (s t) / s<t$ we have $0<C(t)<\pi\left(e^{\lambda}-1\right) / \lambda<3 \pi / 2$.

(ii) $t \geq \pi / \lambda$. Observe that $e^{s} / s$ is decreasing for $s \leq \lambda$. It follows that if we integrate $s^{-1} e^{s} \sin s t$ between successive zeros, these integrals successively alternate in sign and decrease in absolute value. Hence $0<C(t)<e^{\lambda} \int_{0}^{\pi} y^{-1} \sin y d y<\pi e^{\lambda}<3 \pi / 2$.

To conclude this section, we compute the first moment of $h$. 
Theorem 6.9. $\rho_{1}=\int_{0}^{\infty} x h(x) d x=2 e^{\gamma}$, where $\gamma$ is Euler's constant.

Proof. Since $h(x) \rightarrow 0$ rapidly as $x \rightarrow \infty$, we may compute

$$
\rho_{1}=-u^{\prime}(0)=\lim _{z \rightarrow 0}-2 \exp \left(-E_{1}(z)\right) E_{1}^{\prime}(z)\left(1+\exp \left(-E_{1}(z)\right)^{-2} .\right.
$$

Now the result follows from using (6.3).

\section{$\S 7$. Discussion}

In developing the coarsening model considered in this paper, we have been motivated by the study of long time behavior in the nonlinear PDE

$$
u_{\tau}=\varepsilon^{2} u_{x x}+\frac{1}{2}\left(u-u^{3}\right), \quad 0 \leq x \leq 1, \quad \tau \geq 0, \quad u \in \mathbf{R} .
$$

Imposing boundary conditions $u_{x}(0, \tau)=0=u_{x}(1, \tau)$, the only stable states of $(7.1)$ are $u=1$ and $u=-1$. A typical solution converges to one of these as $\tau \rightarrow \infty$, but will develop a pattern of transition layers separating regions where the solution approximates +1 or -1 . These patterns evolve very slowly: Transition layers are approximately described by standing wave solutions. One such is $U(x)=\tanh (x / 2 \varepsilon)$. A transition layer at $x=h$ is approximated by $U(x-h)$ or $U(-x+h)$.

The existence and persistence of patterns of transition layers for (7.1) was established in [3], [4]. Fixing the number $N$ of transition layers and assuming their positions $h_{1}, \ldots, h_{N}$ satisfy $h_{j+1}-h_{j}>C \varepsilon$ for a sufficiently large constant $C$ independent of $\varepsilon$, it was shown that the layer positions evolve in time, satisfying to high accuracy the system of ODEs

$$
\frac{d h_{j}}{d \tau}=12 \varepsilon\left(e^{-\left(h_{j+1}-h_{j}\right) / \varepsilon}-e^{-\left(h_{j}-h_{j-1}\right) / \varepsilon}\right)
$$

with $h_{0}=-h_{1}, h_{N+1}=1+\left(1-h_{N}\right)$. For small $\varepsilon$, the function $x \rightarrow e^{-x / \varepsilon}$ decreases very rapidly with $x$, so it seems reasonable to assume that the system (7.2) is dominated by motion of the layers bounding the smallest interval, while the other layer positions remain essentially unchanged. The length $\ell$ of this smallest interval, if initially equal to $\ell_{0}$, satisfies approximately

$$
\frac{d \ell}{d \tau}=-24 \varepsilon e^{-\ell / \varepsilon}, \quad e^{\ell(\tau) / \varepsilon}=e^{\ell_{0} / \varepsilon}-24 \tau .
$$

This equation predicts that the smallest interval vanishes in a finite time $\tau=O\left(e^{\ell_{0} / \varepsilon}\right)$. Numerically one does observe that transition layers annihilate each other. One then expects the process to be repeated: The smallest interval is eliminated while the others hardly change at all. This is the coarsening process we have modeled in this paper.

Our model of the discrete coarsening process runs on an "intrinsic" time scale proportional to the number of intervals destroyed; recall that $N(t)=N_{0}-2 t$. This time scale may be related to that of the PDE by assuming that the length distribution is self-similar, so that $N(t) L(t)=$ const. It follows that the number of intervals remaining should be approximately proportional to $1 / \varepsilon \ln \tau$. 
The computer simulations in section 3 provide evidence that the ultimate behaviour of our coarsening model is to agree with the self-similar solution of the rate equations. Such agreement should be expected only if the "random order" assumption holds, i.e. the assumption that coalescing intervals are distributed the same as intervals in general. It is clear that this assumption will fail to hold in certain situations of interest. For example, one could imagine that initially intervals are alternately long and short, say with "even" intervals long and "odd" intervals short. Then one can see that the smallest interval is always odd, and the distribution of its neighbors' lengths will not agree with the distribution generally, which is what was assumed in developing equations (2.1)-(2.2). Further work is underway to model such situations, and determine when self-similar coarsening can be expected and when not.

An issue we have not managed to resolve is that of mathematically demonstrating that solutions of the rate equations (2.1)-(2.2) must approach the similarity solution asymptotically in time as $N(t) \rightarrow 0$. To consider this problem, change to similarity variables, writing $f(x, t)=N(t)^{2} g(N x, t), \alpha(t)=N(t) L(t)$. Then $g(w, t)=0$ for $w<\alpha, \int_{0}^{\infty} g(w, t) d t=1$ for $t \geq 0$, and equations (2.1)-(2.2) become

$$
\begin{aligned}
N(t) \frac{d \alpha}{d t}(t) & =-2 \alpha(t)+g(\alpha(t), t)^{-1} \\
N(t) g_{t}(w, t)-2 w g_{w}(w, t) & =2 g(w, t)+\int_{0}^{\infty} g(y, t) g(w-y-\alpha(t), t) d y
\end{aligned}
$$

We expect as $t \rightarrow N_{0} / 2$ that $N(t) g_{t} \rightarrow 0$ and $\alpha(t) \rightarrow \alpha>0$ consistent with $g(w, t) \rightarrow g(w)$ where $g(w)$ satisfies (2.7). One possible strategy to establish this is to study the Laplace transform $G(z, t)=\int_{0}^{\infty} e^{-w z} g(w, t) d w$. It follows from (7.4) that $G$ satisfies

$$
N(t) G_{t}(z, t)+2 z G_{z}(z, t)=e^{-\alpha(t) z}\left(G(z, t)^{2}-1\right)
$$

This equation may be studied by characteristics to obtain a formula for $G(z, t)$ in terms of $G(z, 0)$ and $\alpha(t)$, namely

$$
\tanh ^{-1} G(z, t)=-\int_{0}^{t} N(s)^{-1} e^{-z N(t) L(s)} d s+\tanh ^{-1} G\left(z N(t) / N_{0}, 0\right)
$$

If one could prove that $\alpha(t)$ converges as $t \rightarrow N_{0} / 2$, then one could exploit this formula to prove that $G(z, t)$ converges to the appropriate limit. Unfortunately we have not been able to show that $\alpha(t)$ converges; we only know from Lemma 4.4 that $\alpha(t)<\rho$ for all $t$. 


\section{References}

1. M. Abramowitz and I.A. Stegun, Handbook of Mathematical Functions, Dover, 1972.

2. J. M. Ball and J. Carr, The discrete coagulation-fragmentation equations: existence, uniqueness, and density conservation, J. Stat. Phys. 61 (1990) 203-234.

3. J. Carr and R.L. Pego, Metastable patterns in solutions of $u_{t}=\varepsilon^{2} u_{x x}-f(u)$, Comm. Pure Appl. Math. 42 (1989) 523-576.

4. J. Carr and R.L. Pego, Invariant manifolds for metastable patterns in $u_{t}=\varepsilon^{2} u_{x x}-f(u)$. Proc. Royal Soc. Edinburgh, 116A (1990) 133-160.

5. O. Diekmann and H.G. Kaper, On the bounded solutions of a nonlinear convolution equation, Nonlin. Anal. 2 (1978) 721-737.

6. M. H. Ernst, Exact solutions of the nonlinear Boltzmann equation and related kinetic equations, in Studies in Statistical Mechanics, vol X, eds. E.W. Montroll and J.L. Lebowitz, North -Holland, 1983.

7. W. Feller, An Introduction to Probability Theory and its Applications, Vol 2 (second edition), Wiley, 1966.

8. F.W.J. Olver, Asymptotics and Special Functions, Academic Press, 1974.

9. P.W. Voorhees, The theory of Ostwald ripening, J. Stat. Phys. 38 (1985) 231-252.

10. D.V. Widder, The Laplace Transform, Princeton University Press, 1941. 


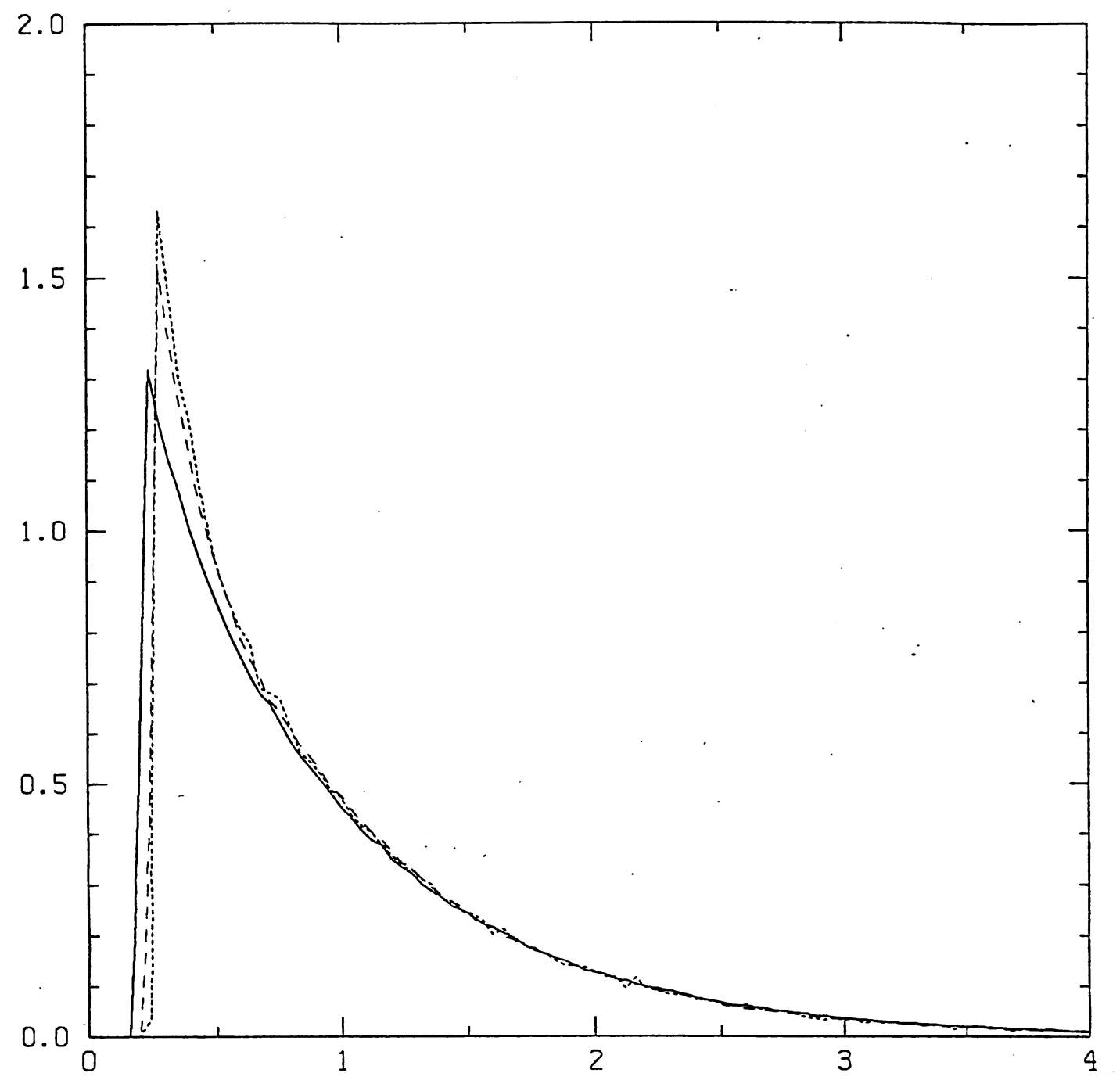

Fig 1a. Simulation data for case (a) 


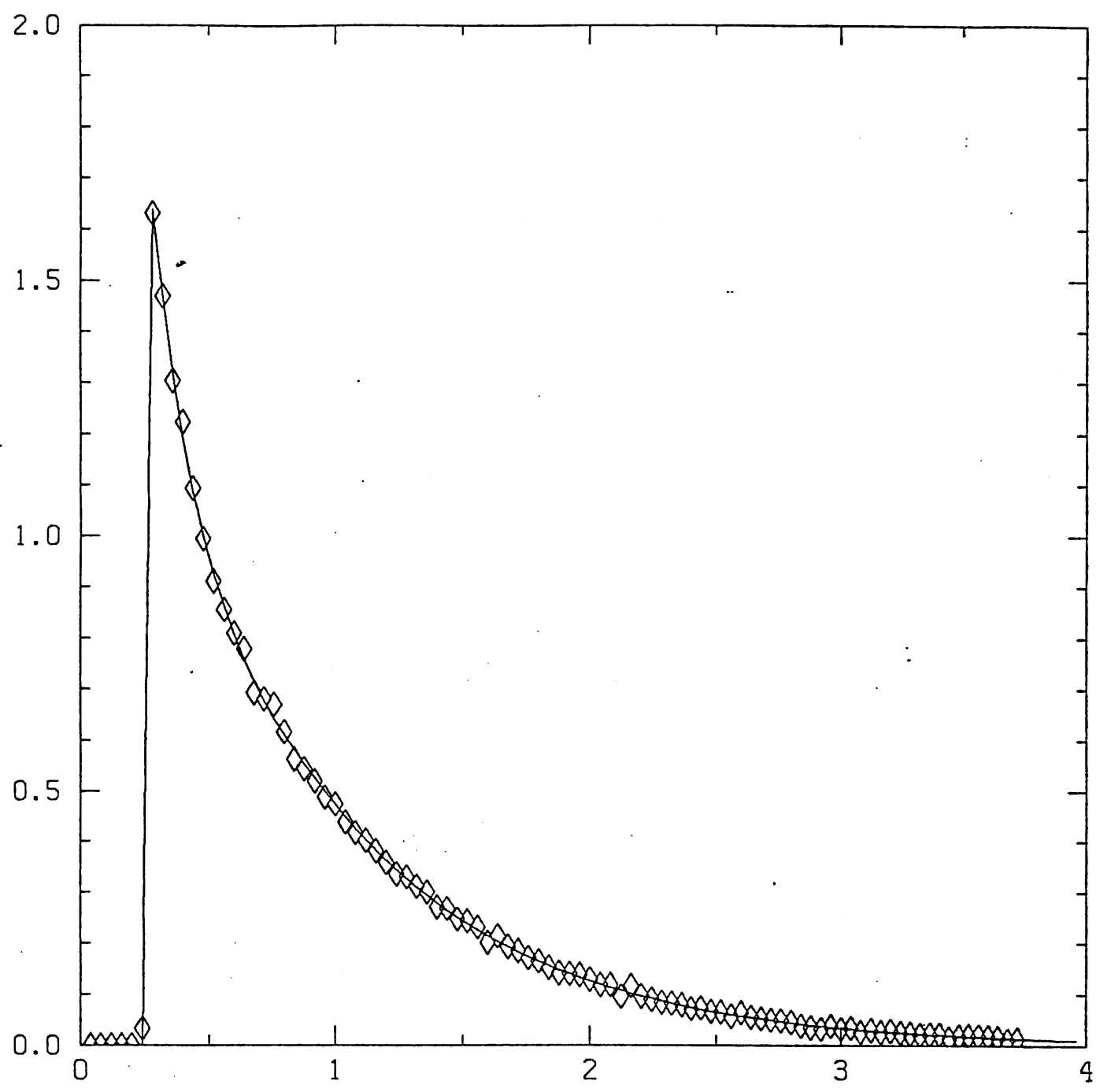

Fig 1b. Similarity solution compared to simulation for case (a) 


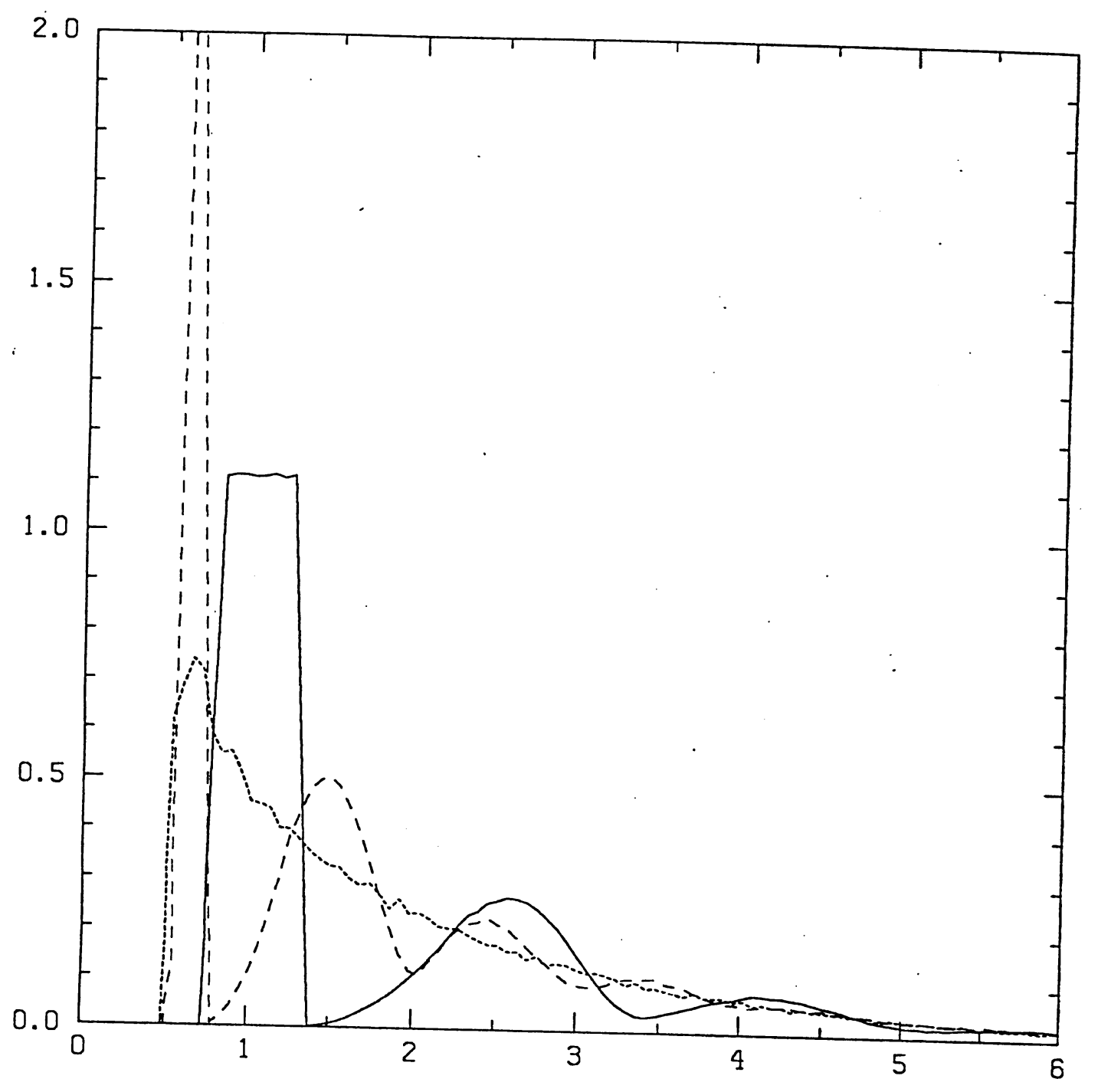

Fig 2a. Simulation data for case (b) 


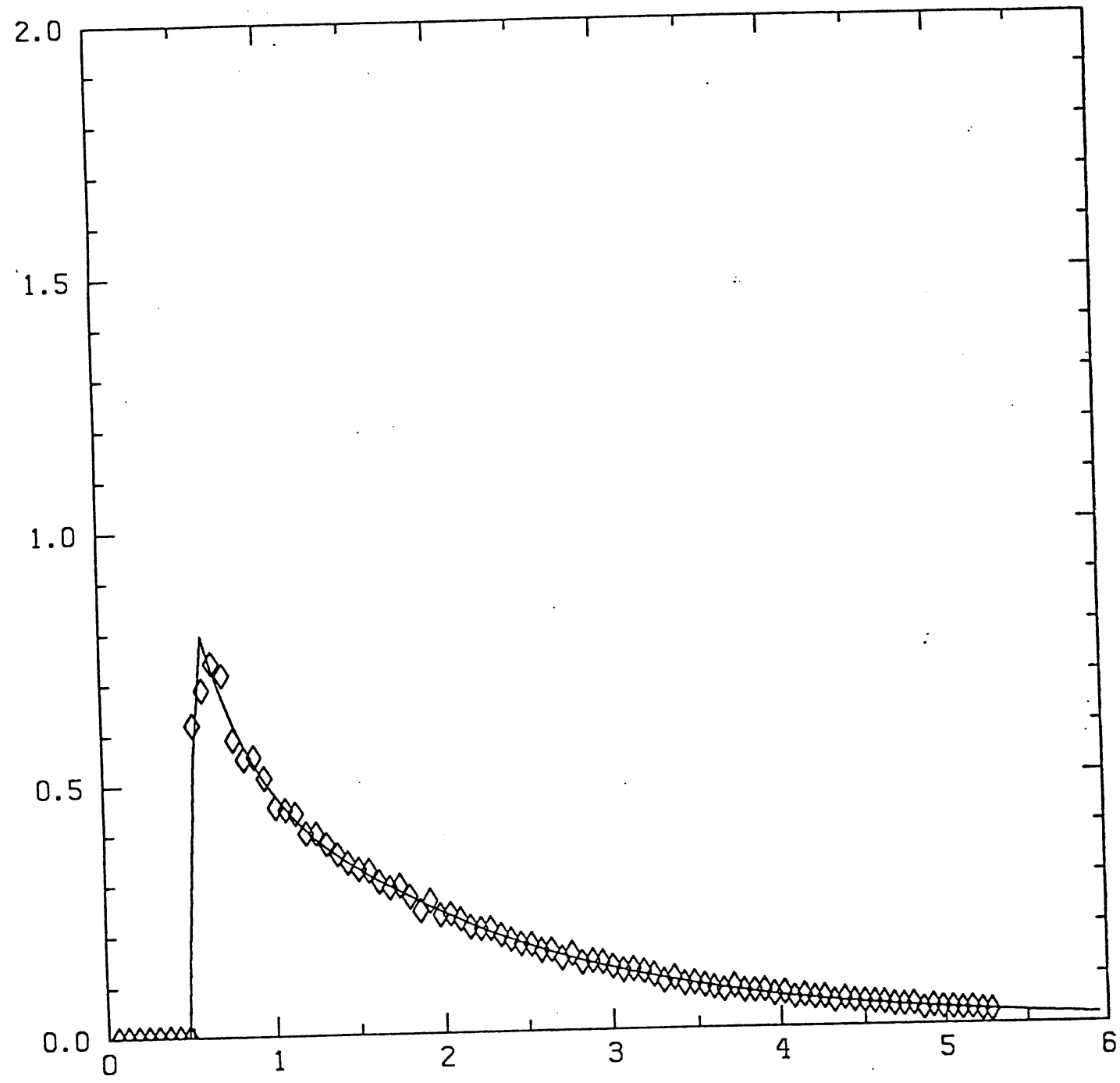

Fig 2b. Similarity solution compared to simulation for case (b) 


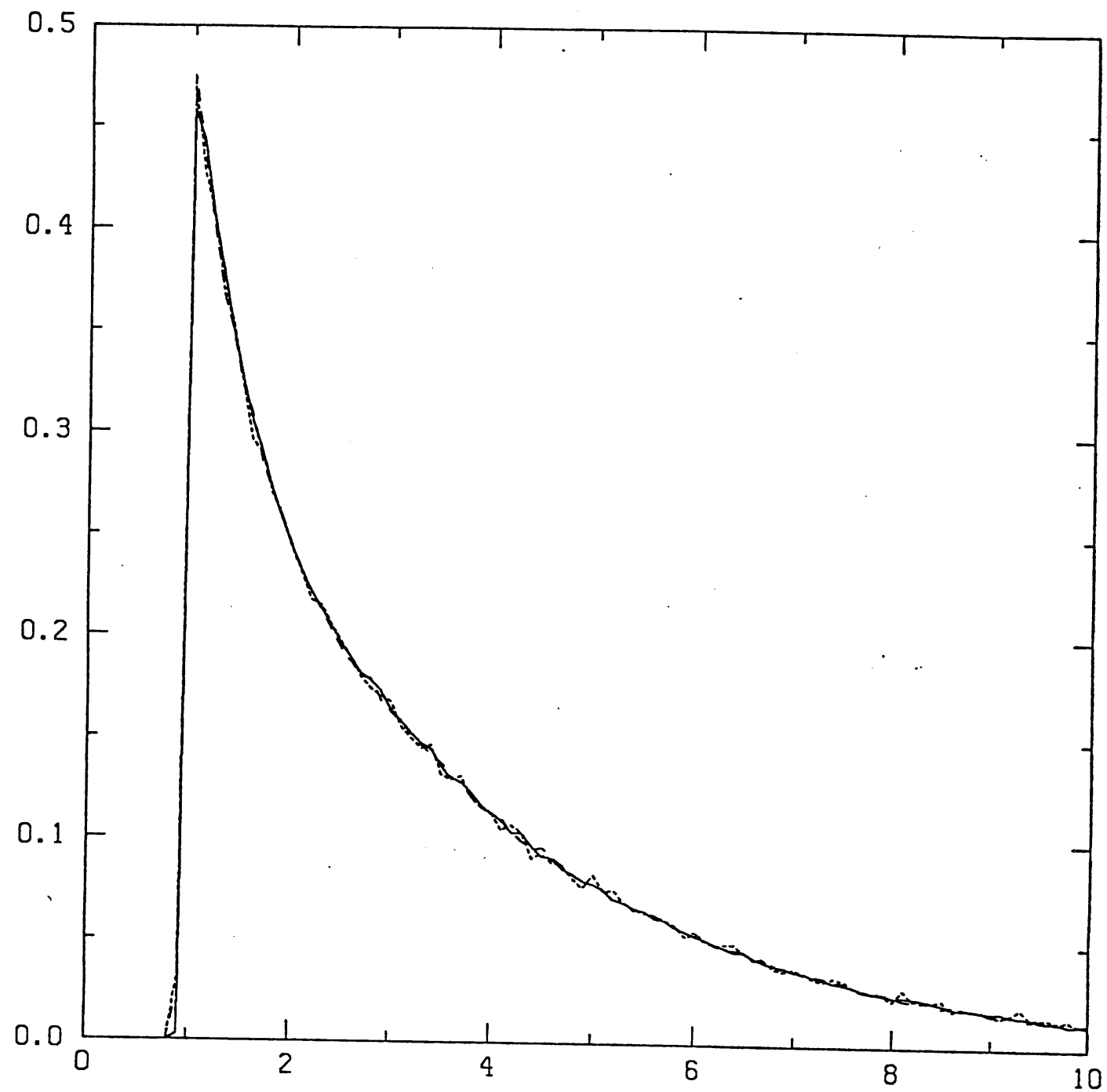

Fig 3a. Simulation data for case (c) 


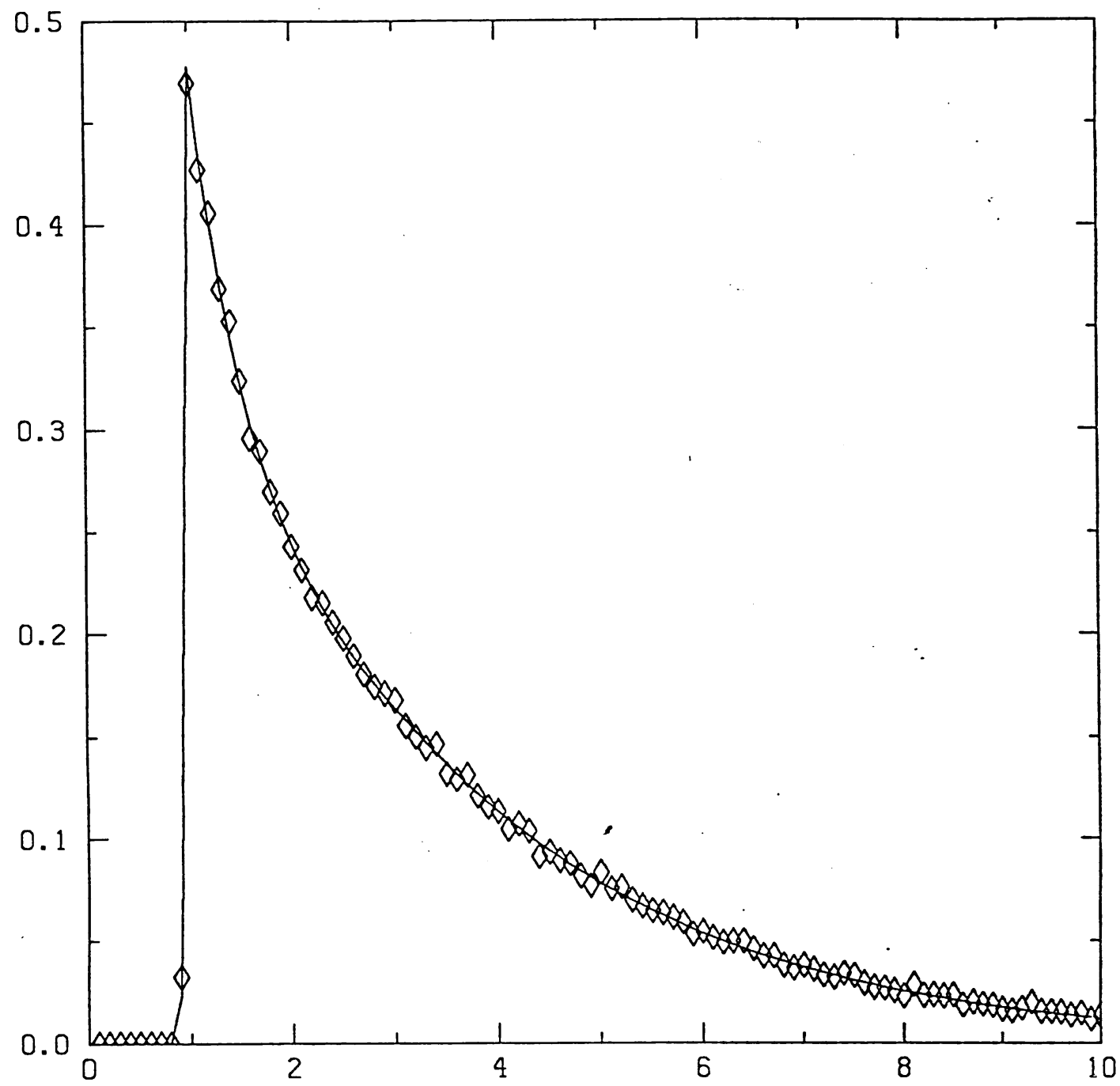

Fig 3b. Similarity solution compared to simulation for case (c) 

equations with damping

A. Eden, C. Foias, B. Nicolaenko \& R. Temam, Inertial sets for dissipative evolution equations

A. Eden, C. Foias, B. Nicolaenko \& R. Temam, Hölder continuity for the inverse of Mañé's projection

Michel Chipot and Charles Collins, Numerical approximations in variational problems with potential wells

Huanan Yang, Nonlinear wave analysis and convergence of MUSCL schemes

László Gerencsér and Zsuzsanna Vágó, A strong approximation theorem for estimator processes in continuous time

László Gerencsér, Multiple integrals with respect to $L$-mixing processes

David Kinderlehrer and Pablo Pedregal, Weak convergence of integrands and the Young measure representation

Bo Deng, Symbolic dynamics for chaotic systems

P. Galdi, D.D. Joseph, L. Preziosi, S. Rionero, Mathematical problems for miscible, incompressible fluids with Korteweg stresses

Charles Collins and Mitchell Luskin, Optimal order error estimates for the finite element approximation of the solution of a nonconvex variational problem

Peter Gritzmann and Victor Klee, Computational complexity of inner and outer j-radii of polytopes in finite-dimensional normed spaces

A. Ronald Gallant and George Tauchen, A nonparametric approach to nonlinear time series analysis: estimation and simulation

H.S. Dumas, J.A. Ellison and A.W. Sáenz, Axial channeling in perfect crystals, the continuum model and the method of averaging

M.A. Kaashoek and S.M. Verduyn Lunel, Characteristic matrices and spectral properties of evolutionary systems

Xinfu Chen, Generation and Propagation of interfaces in reaction diffusion systems

Avner Friedman and Bei Hu, Homogenization approach to light scattering from polymer-dispersed liquid crystal films

Yoshihisa Morita and Shuichi Jimbo, ODEs on inertial manifolds for reaction-diffusion systems in a singularly perturbed domain with several thin channels

Wenxiong Liu, Blow-up behavior for semilinear heat equations:multi-dimensional case

Hi Jun Choe, Hölder continuity for solutions of certain degenerate parabolic systems

Hi Jun Choe, Regularity for certain degenerate elliptic double obstacle problems

Fernando Reitich, On the slow motion of the interface of layered solutions to the scalar Ginzburg-Landau equation

Xinfu Chen and Fernando Reitich, Local existence and uniqueness of solutions of the Stefan problem with surface tension and kinetic undercooling

C.C. Lim, J.M. Pimbley, C. Schmeiser and D.W. Schwendeman, Rotating waves for semiconductor inverter rings

W. Balser, B.L.J. Braaksma, J.-P. Ramis and Y. Sibuya, Multisummability of formal power series solutions of linear ordinary differential equations

Peter J. Olver and Chehrzad Shakiban, Dissipative decomposition of partial differential equations Clark Robinson, Homoclinic bifurcation to a transitive attractor of Lorenz type, II

Michelle Schatzman, A simple proof of convergence of the $Q R$ algorithm for normal matrices without shifts Ian M. Anderson, Niky Kamran and Peter J. Olver, Internal, external and generalized symmetries C. Foias and J.C. Saut, Asymptotic integration of Navier-Stokes equations with potential forces. I Ling Ma, The convergence of semidiscrete methods for a system of reaction-diffusion equations Adelina Georgescu, Models of asymptotic approximation

A. Makagon and H. Salehi, On bounded and harmonizable solutions on infinite order arma systems San-Yih Lin and Yan-Shin Chin, An upwind finite-volume scheme with a triangular mesh for conservation laws J.M. Ball, P.J. Holmes, R.D. James, R.L. Pego \& P.J. Swart, On the dynamics of fine structure KangPing Chen and Daniel D. Joseph, Lubrication theory and long waves

J.L. Ericksen, Local bifurcation theory for thermoelastic Bravais lattices

Mario Taboada and Yuncheng You, Some stability results for perturbed semilinear parabolic equations

A.J. Lawrance, Local and deletion influence

Bogdan Vernescu, Convergence results for the homogenization of flow in fractured porous media

Xinfu Chen and Avner Friedman, Mathematical modeling of semiconductor lasers

Yongzhi Xu, Scattering of acoustic wave by obstacle in stratified medium 
system part I: the Riemann problem

Epifanio G. Virga, New variational problems in the statics of liquid crystals

Yoshikazu Giga and Shun'ichi Goto, Geometric evolution of phase-boundaries

Ling Ma, Large time study of finite element methods for 2D Navier-Stokes equations

Mitchell Luskin and Ling Ma, Analysis of the finite element approximation of microstructure in micromagnetics

M. Chipot, Numerical analysis of oscillations in nonconvex problems

J. Carrillo and M. Chipot, The dam problem with leaky boundary conditions

Eduard Harabetian and Robert Pego, Efficient hybrid shock capturing schemes

B.L.J. Braaksma, Multisummability and Stokes multipliers of linear meromorphic differential equations

Tae Il Jeon and Tze-Chien Sun, A central limit theorem for non-linear vector functionals of vector Gaussian processes

Chris Grant, Solutions to evolution equations with near-equilibrium initial values

Mario Taboada and Yuncheng You, Invariant manifolds for retarded semilinear wave equations

Peter Rejto and Mario Taboada, Unique solvability of nonlinear Volterra equations in weighted spaces

Hi Jun Choe, Holder regularity for the gradient of solutions of certain singular parabolic equations

Jack D. Dockery, Existence of standing pulse solutions for an excitable activator-inhibitory system

Jack D. Dockery and Roger Lui, Existence of travelling wave solutions for a bistable evolutionary ecology model

Giovanni Alberti, Luigi Ambrosio and Giuseppe Buttazzo, Singular perturbation problems with a compact support semilinear term

Emad A. Fatemi, Numerical schemes for constrained minimization problems

Y. Kuang and H.L. Smith, Slowly oscillating periodic solutions of autonomous state-dependent delay equations

Emad A. Fatemi, A new splitting method for scaler conservation laws with stiff source terms

Hi Jun Choe, A regularity theory for a more general class of quasilinear parabolic partial differential equations and variational inequalities

Haitao Fan, A vanishing viscosity approach on the dynamics of phase transitions in Van Der Waals fluids

T.A. Osborn and F.H. Molzahn, The Wigner-Weyl transform on tori and connected graph propagator representations

Avner Friedman and Bei Hu, A free boundary problem arising in superconductor modeling

Avner Friedman and Wenxiong Liu, An augmented drift-diffusion model in semiconductor device

Avner Friedman and Miguel A. Herrero, Extinction and positivity for a system of semilinear parabolic variational inequalities

David Dobson and Avner Friedman, The time-harmonic Maxwell equations in a doubly periodic structure

Hi Jun Choe, Interior behaviour of minimizers for certain functionals with nonstandard growth

Vincenzo M. Tortorelli and Epifanio G. Virga, Axis-symmetric boundary-value problems for nematic liquid crystals with variable degree of orientation

Nikan B. Firoozye and Robert V. Kohn, Geometric parameters and the relaxation of multiwell energies

Haitao Fan and Marshall Slemrod, The Riemann problem for systems of conservation laws of mixed type

Joseph D. Fehribach, Analysis and application of a continuation method for a self-similar coupled Stefan system

C. Foias, M.S. Jolly, I.G. Kevrekidis and E.S. Titi, Dissipativity of numerical schemes
D.D. Joseph, T.Y.J. Liao and J.-C. Saut, Kelvin-Helmholtz mechanism for side branching in the displacement of light with heavy fluid under gravity

Chris Grant, Solutions to evolution equations with near-equilibrium initial values

B. Cockburn, F. Coquel, Ph. LeFloch and C.W. Shu, Convergence of finite volume methods

N.G. Lloyd and J.M. Pearson, Computing centre conditions for certain cubic systems

João Palhoto Matos, Young measures and the absence of fine microstructures in the $\alpha-\beta$ quartz phase transition

L.A. Peletier \& W.C. Troy, Self-similar solutions for infiltration of dopant into semiconductors

H. Scott Dumas and James A. Ellison, Nekhoroshev's theorem, ergodicity, and the motion of energetic charged particles in crystals

Stathis Filippas and Robert V. Kohn, Refined asymptotics for the blowup of $u_{t}-\Delta u=u^{p}$.

Patricia Bauman, Nicholas C. Owen and Daniel Phillips, Maximum principles and a priori estimates for an incompressible material in nonlinear elasticity

Patricia Bauman, Nicholas C. Owen and Daniel Phillips, Maximal smoothness of solutions to certain Euler-Lagrange equations from nonlinear elasticity

Jack Carr and Robert Pego, Self-similarity in a coarsening model in one dimension

J.M. Greenberg, The shock generation problem for a discrete gas with short range repulsive forces

George R. Sell and Mario Taboada, Local dissipativity and attractors for the Kuramoto-Sivashinsky equation in thin $2 \mathrm{D}$ domains

T. Subba Rao, Analysis of nonlinear time series (and chaos) by bispectral methods 